\title{
DESEMPEÑO LABORAL EN EMPRESAS PRIVADAS DE CONTADORES FORMADOS EN UNIVERSIDADES MANABITAS
}

\author{
WORK PERFORMANCE IN ACCOUNTANT PRIVATE COMPANIES FORMED IN \\ MANABI UNIVERSITIES \\ RAFAel Bermúdez TACUngA* \\ Profesor Titular Principal de la Escuela de Contabilidad y Auditoría \\ Universidad Laica Eloy Alfaro de Manabí (ULEAM) / Ecuador \\ [Recepción: Febrero de 2015 / Conformidad: Abril de 2015]
}

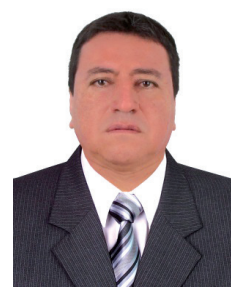

\section{RESUMEN}

En el presente artículo se ha efectuado un análisis sobre la formación profesional que recibieron los contadores dentro de las universidades manabitas, los que mantienen actividades laborales con empresas que funcionan dentro de la misma provincia, debido a que generalmente existe una evidente contradicción entre la educación superior que brindan las universidades y las exigencias que requiere una empresa de un profesional contratado con respecto a los conocimientos, habilidades y valores que posee para el ejercicio de sus funciones. La investigación realizada fue de tipo explorativa y descriptiva; la metodología adoptada en la investigación para la recolección de la información a través de correo electrónico, se ha realizado mediante la formulación de un cuestionario con preguntas cerradas relacionadas con la formación recibida en las universidades y su aplicabilidad en las funciones que desempeña; se asumió una muestra de 40 contadores a quienes se les aplicó el instrumento para determinar el nivel de satisfacción de la formación recibida en la institución de educación superior. Entre los resultados más relevantes obtenidos y de los cuales se derivaron las conclusiones expuestas en el artículo, se destacan ciertos aspectos de inconformidad demostrada por los encuestados respecto al uso de tecnología en su etapa formativa universitaria.

\section{Palabras clave:}

Desempeño laboral; empresas privadas; contadores; universidades.

\begin{abstract}
In this article we made an analysis of the professional training the accountants received in universities from Manabi, they maintain working activities with companies operating within the same province, since there is usually an obvious contradiction between the higher education offered by universities and the requirements an enterprise needs of a hired professional regarding the knowledge, skills and values that they have for the exercise of their functions. The research was exploratory and descriptive; the methodology adopted in the research for the collection of information via email, was made by developing a questionnaire with closed questions about the training in universities and their applicability in the functions performed; a sample of 40 accountants were handed the instrument to determine the level of satisfaction with the training received in higher education institutions. Among the most relevant results and of which the conclusions in the article were derived, we highlight certain aspects of dissatisfaction shown by respondents regarding the use of technology in their university training.
\end{abstract}

\section{Keywords:}

Work performance; private businesses; accountants; universities.

* Ingeniero Comercial -ULEAM/ Ecuador. Email: rafabermudez12@yahoo.com 


\section{INTRODUCCIÓN}

Las universidades ecuatorianas y por ende, las universidades de la provincia de Manabí1 ${ }^{1}$, tienen el encargo social de la formación de profesionales que posean todas las capacidades y actitudes necesarias para que puedan desarrollar nuevas actividades productivas empresariales o que puedan aportar con sus funciones con el eficiente desarrollo económico de las empresas que requieren de sus servicios. Al respecto, la Organización de las Naciones Unidas para la Educación, la Ciencia y la Cultura (UNESCO) establece que una de las misiones y funciones de las universidades es "formar diplomados altamente cualificados y ciudadanos responsables, capaces de atender a las necesidades de todos los aspectos de la actividad humana, ofreciéndoles cualificaciones que estén a la altura de los tiempos modernos, comprendida la capacitación profesional, en las que se combinen los conocimientos teóricos y prácticos de alto nivel mediante cursos y programas que estén constantemente adaptados a las necesidades presentes y futuras de la sociedad"2.

Otro importante aporte sobre las funciones contemporáneas de la universidades es la emitida por Pelikan (1992), quien señala que unas de las labores que se desarrollan en la universidad moderna son: el avance del conocimiento a través de la investigación; la extensión del conocimiento a través de la enseñanza a nivel de licenciatura y de posgrado; el entrenamiento que comprende tanto conocimientos como habilidades en las escuelas profesionales de la universidad; la preservación del conocimiento en bibliotecas, galerías y museos y, la difusión del conocimiento a través de publicaciones académicas. Además, el citado autor agrega que "una universidad puede hacer muchas otras cosas, y puede hacer todas ellas simultáneamente. También, hay cosas adicionales que una universidad debe hacer como parte de sus obligaciones con la sociedad, las cuales cambiarán de una sociedad a otra, o de un momento histórico a otro, y de una universidad a otra; pero las enunciadas anteriormente son las cosas que debe estar haciendo, si quiere ser una Universidad" (p. 76).

En base a lo expuesto por la UNESCO y por Pelikan en los párrafos anteriores, se puede establecer que la universidad tiene una responsabilidad importante con el desarrollo socio económico de una sociedad, la cual se vuelve objetiva mediante varios productos derivados de las acciones educativas que la organización ejecuta en los estudiantes, uno de estos productos destacable es la formación integral de profesionales que requieren las empresas para el cumplimiento de funciones técnicas, en el caso específico del presente estudio, profesionales expertos en conocimientos y habilidades contables que le permitan ser un miembro de la empresa con cabal cumplimiento de acciones aplicativas, analíticas e interpretativas de toda la información contable y financiera que se genere por efectos de la actividad económica que ejecuta la organización para la cual labora, con la principal finalidad de que la alta dirección puedan tener la información necesaria que le permita tomar decisiones más acertadas en beneficio de los intereses de la organización; el contador debe convertirse en un verdadero apoyo y asesor de la alta gerencia, por lo que, actualmente debe asumir funciones administrativas que le requieren capacidades en funciones que van más allá del análisis numérico y estructuración de informes contables elaborados mediante la utilización de programas y tecnología contemporánea. $\mathrm{Al}$ respecto, Montaner y Perozo (2008), en el artículo titulado "Formación ética del Contador Público. Caso: Facultad de Ciencias Económicas y Sociales de la Universidad del Zulia” ${ }^{3}$ consideran que el Contador Público debe ser un profesional con habilidades y actitudes necesarias para promover dentro de las organizaciones un desarrollo económico a través de la producción, análisis e interpretación de diversos informes financieros. Sin embargo, en ocasiones estos profesionales tergiversan sus actividades convirtiéndose en instrumentos de manejos dolosos.

\footnotetext{
1 Manabí es una provincia ecuatoriana localizada en el emplazamiento centro-noroeste del Ecuador continental.

2 Conferencia Mundial sobre la Educación Superior. La educación superior en el siglo XXI: Visión y acción. 9 de octubre de 1998.

3 Disponible en: http://www.scielo.org.ve/scielo.php?pid=S1315-95182008000200013\&script=sci_arttext
} 
Por otro lado, el Instituto de Investigaciones Contables del Ecuador $\left(\mathrm{IICE}^{4}\right)$ y la Federación Nacional de Contadores del Ecuador (FNCE), en su Código de Ética del Contador, consideran ciertos postulados generales que deben regir la conducta de un contador en sus relaciones profesionales con empresas de cualquier índole o en el desempeño profesional independiente; entre los postulados más importantes se pueden citar: a) Integridad: Un contador debe ser recto, honesto y sincero al realizar su trabajo profesional; b) Objetividad: Un contador debe ser imparcial y no permitir que el prejuicio o la predisposición contrarresten su objetividad cuando emita su opinión profesional respecto de los estados financieros revisados por él, deberá mantener una actitud imparcial; c) Normas Técnicas: Un contador deberá efectuar su trabajo profesional con cuidado y eficiencia, de acuerdo con las normas técnicas y profesionales relativas para dicho trabajo, promulgadas por el IICE y en concordancia con la legislación ecuatoriana, siempre y cuando tales instrucciones no sean incompatibles con los requisitos de integridad, objetividad e independencia; d) Capacidad Profesional: Un contador tiene la obligación de mantener su nivel de competencia a lo largo de toda su carrera profesional. Solamente deberá aceptar trabajos que él o su firma esperen poder realizar con capacidad profesional.

El tema del presente trabajo investigativo se ha escogido por cuanto es necesario efectuar permanentemente un análisis de la correlación que existe entre la demanda que efectúan las empresas privadas de las capacidades que debe poseer un profesional en contabilidad para su eficiente desempeño profesional dentro de las organizaciones, y la calidad de formación que las universidades vienen desarrollando en sus estudiantes; esta relación es un elemento fundamental para la determinación de la pertinencia de las carreras que ofertan las instituciones de educación superior dentro de una región. En Ecuador, actualmente se viene incorporando un proceso de cambio de la matriz productiva mediante la cual, el gobierno ecuatoriano, viene planteando la transformación de las actividades económicas de la mayoría de las empresas ecuatorianas, y pasar de ser productores permanentes de materias primarias a ser fabricantes de productos terminados con alto valor agregado; esta situación, de manera contundente, exige también que los profesionales que egresan de las universidades tengan un alto nivel competitivo para satisfacer las expectativas de las modernas empresas, y por ende, demanda que las universidades incrementen la calidad formativa y educativa que ofrecen; no es posible mejorar la competitividad del sector productivo empresarial, sin mejorar los profesionales que forman parte de la organización. Dentro de estos profesionales se encuentran los contadores formados en las universidades de la provincia de Manabí, por lo que resulta importante que se hagan investigaciones periódicas y permanentes de las capacidades adquiridas por los profesionales dentro de las universidades y sus aplicaciones dentro del sector empresarial privado de la provincia; en lo expuesto anteriormente radica la importancia de la investigación efectuada y que se expone en el presente artículo.

El estudio del desempeño laboral dentro de las empresas de los profesionales que forman las universidades, es un aspecto importante que constantemente debe vigilarse para efectos de evaluar si la calidad educativa superior en un país o región es la adecuada para el alcance de los propósitos productivos, económicos y sociales que posee un Estado, lo cual es un indicador del cumplimiento de la responsabilidad social que poseen las instituciones de educación superior. Al respecto, Vallaeys (2008), en su artículo ¿Qué es la Responsabilidad Social Universitaria ${ }^{5}$, establece que mediante el cumplimiento de la responsabilidad social de las universidades "no sólo se trata de reformar a las malas políticas del estado, sino también a los malos conocimientos y epistemologías que la Universidad contribuye en producir y transmitir, y que inducen estas malas políticas, cada Universidad podrá empezar a elaborar su propio diagnóstico y reforma. La Responsabilidad Social Universitaria exige,

\footnotetext{
4 El Instituto de Investigaciones Contables del Ecuador (IICE), es el Órgano Técnico de la Profesión de Contaduría Pública y voz autorizada de la Federación Nacional de Contadores del Ecuador, en el aspecto técnico. Establecido con Estatuto aprobado en el Ministerio de Educación Pública, mediante Acuerdo Ministerial Nº. 2002 del 8 de julio de 1964.

5 Disponible en: http://www.unitru.edu.pe/Publicaciones/Jornada/Responsabilidad_Social_Universitaria.pdf
} 
desde una visión holística, articular las diversas partes de la institución en un proyecto de promoción social de principios éticos y de desarrollo social equitativo y sostenible, para la producción y transmisión de saberes responsables y la formación de profesionales ciudadanos igualmente responsables".

El objetivo de la investigación efectuada fue relacionar los conocimientos, habilidades y valores que las universidades transmiten a sus estudiantes de contabilidad, para efectos de que su desempeño laboral profesional sea el más eficiente posible dentro de las empresas privadas de diferentes actividades económicas. El objetivo planteado tiene relación con el desempeño laboral de un profesional, el cual según Bohórquez (2002) es el nivel de ejecución alcanzado por el trabajador en el logro de las metas dentro de la organización en un tiempo determinado (citado en Araujo y Guerra, 2007). Otra definición importante es la que usa Chiavenato (2002), quien expone que el desempeño es eficacia del personal que trabaja dentro de las organizaciones, la cual es necesaria para la organización, funcionando el individuo con una gran labor y satisfacción laboral (citado en Araujo y Guerra, 2007). En las definiciones expuestas anteriormente se pueden destacar dos elementos presentes en el desempeño laboral, el primero es la actitud y aptitud que tiene el trabajador y el segundo, son los resultados que se alcanzan dentro de la empresa, los cuales se evalúan en función de los objetivos que la organización se haya planteado durante la etapa de planeación.

También otro aporte importante en la conceptualización del desempeño laboral es dada por Stoner (1994), quien afirma que el "desempeño laboral es la manera como los miembros de la organización trabajan eficazmente, para alcanzar metas comunes, sujeto a las reglas básicas establecidas con anterioridad"; al igual que el autor anterior, en esta definición se establece una relación del trabajo eficiente con el alcance de los fines de la organización; este trabajo eficiente que expone Stoner, en el caso de los contadores profesionales graduados en las universidades, debe ser desarrollado paulatinamente en las aulas y áreas de estudio que tengan las universidades para tal efecto y demostrado objetivamente en las empresas que requieran de sus servicios.
Por otro lado, en esta investigación se expone como beneficiario del desempeño laboral demostrado por los contadores, a las empresas privadas de la provincia de Manabí, por lo cual es necesario definir esta variable principal empleada en el artículo; según García y Casanueva (2001) la empresa es "una entidad que mediante la organización de elementos humanos, materiales, técnicos y financieros proporciona bienes o servicios a cambio de un precio que le permite la reposición de los recursos empleados y la consecución de unos objetivos determinados".

Como se puede apreciar, en el concepto de empresa expuesto anteriormente se hace alusión a empleo de recursos, entre los cuales se encuentra el talento humano, con la finalidad de producir y alcanzar metas, las cuales generalmente tienen relación con la obtención de márgenes de utilidades que le permitan crecer en los mercados y ser altamente competitivos dentro de los mismos. Dentro de estos recursos humanos, se encuentran los contadores que forman parte de la estructura administrativa financiera de las empresas y que con su desempeño, coordinado con el trabajo de otros empleados, permiten el alcance eficiente de los objetivos empresariales.

Todas las argumentaciones expuestas en los párrafos anteriores, justifican plenamente la realización de investigaciones que permitan tener una evaluación de la satisfacción que tienen las empresas privadas de una determinada región respecto del trabajo que efectúan sus respectivos contadores. Los resultados obtenidos en el presente trabajo investigativo servirá como referencia para futuras investigaciones que se efectúen sobre temas similares, y en el caso de ser efectuadas en otro entorno diferente al ecuatoriano, se podrán asumir comparaciones entre resultados, de los cuales se derivarán nuevas hipótesis que permitirán noveles aportes para las ciencias. Además, los resultados obtenidos en esta investigación, sirven para que las universidades de la provincia de Manabí y otras regiones del Ecuador, e inclusive otras regiones del continente, puedan plantearse interrogantes sobre la calidad de los profesionales contadores que están formado dentro de su claustro, considerando en todo momento, que las capacidades que demandan las empresas de sus empleados no son estáticas, estas cambian en función de las exigencias competitivas

24/ QVIPURAMAYOC | Vol. 23(43) 2015 
del mercado y en relación a los grandes avances tecnológicos que tiene actualmente el entorno mundial.

Una limitación existente en el estudio efectuado está determinado por el número de la muestra que se asumió, por lo que el presente trabajo no pretende ser una representación técnica de lo que sucede en el país, sino que únicamente es una descripción y análisis de la opinión de contadores sobre su satisfacción de la formación obtenida en las universidades, luego de haber tenido la oportunidad de demostrar sus capacidades a través del desempeño laboral dentro de alguna empresa privada; igualmente, otra limitación es el hecho de que durante la investigación se asume únicamente el criterio de los profesionales contadores que laboran en las empresas privadas, $y$ no considera la opinión de los empleadores, lo cual, sin duda alguna, limita el análisis cruzado de las opiniones para efectos de tener una mejor consideración de la satisfacción tanto del profesional como de los directivos de las organizaciones.

\section{RESULTADOS}

A la muestra establecida de contadores y que fueron seleccionados de manera aleatoria simple de un listado del colegio de contadores de la provincia de Manabí, se les solicitó a través de sus respectivos correos electrónicos que respondieran a un cuestionario estructurado de preguntas, para esto, previamente se evidenció de que el contador seleccionado tenga una relación laboral, al momento de la encuesta con alguna empresa dentro de la provincia; los resultados obtenidos se exponen en los cuadros que siguen posteriormente y sobre los cuales se efectúa un análisis pertinente:

\section{Cuadro No 01:}

¿Cuántos años tiene laborando en la empresa?

\begin{tabular}{|c|r|r|}
\hline Alternativas & \multicolumn{1}{|c|}{ fa } & \multicolumn{1}{|c|}{ f\% } \\
\hline 5 o menos & 8 & 20 \\
\hline Más de 5 & 32 & 80 \\
\hline Total & $\mathbf{4 0}$ & $\mathbf{1 0 0}$ \\
\hline
\end{tabular}

Fuente: Elaboración propia.
Mediante la primera pregunta se buscó establecer la cantidad de años de experiencia que tuvieron los contadores encuestados, lo cual es un indicador importante para estimar la relevancia que tuvieran las opiniones vertidas por ellos; así se tiene que el $80 \%$ de los encuestados mantienen relaciones laborales con sus respectivas empresas por un tiempo mayor de 5 años, únicamente el 20\% restante tiene 5 o menos años de labores activas en la organización.

\section{Cuadro No 02:}

¿Fue complicado encontrar trabajo relacionado con su profesión luego de la culminación de sus estudios?

\begin{tabular}{|c|r|r|}
\hline Alternativas & \multicolumn{1}{|c|}{ fa } & \multicolumn{1}{|c|}{ f\% } \\
\hline Mucho & 36 & 90 \\
\hline Poco & 2 & 5 \\
\hline Nada & 2 & 5 \\
\hline Total & $\mathbf{4 0}$ & $\mathbf{1 0 0}$ \\
\hline
\end{tabular}

Fuente: Elaboración propia.

El 90\% de los contadores tuvieran altos inconvenientes para poder ingresar a las empresas a desempeñar sus actividades profesionales, estos problemas para el ingreso laboral se convierte en un factor que genera una percepción negativa sobre la decisión de haber seguido una carrera profesional de contador en las universidades; en la investigación efectuada se pudo evidenciar que los inconvenientes surgen generalmente por el elevado número de profesionales contadores que existen en la provincia, mismos que se convierten en un exceso de oferta en relación a la cantidad de empresas que existen, inconveniente que se evidencia mucho más en las ciudades pequeñas donde existe poco desarrollo de las medianas y grandes empresas, mismas que son las que requieren mayormente de mano de obra calificada.

\section{Cuadro No 03:}

¿Se encuentra satisfecho con la remuneración que percibe en la empresa?

\begin{tabular}{|c|r|r|}
\hline Alternativas & \multicolumn{1}{|c|}{ fa } & \multicolumn{1}{|c|}{ f\% } \\
\hline $\mathrm{Si}$ & 2 & 5 \\
\hline $\mathrm{No}$ & 38 & 95 \\
\hline Total & $\mathbf{4 0}$ & $\mathbf{1 0 0}$ \\
\hline
\end{tabular}

Fuente: Elaboración propia. 
Existe un elevado número de contadores profesionales que no están conformes con la remuneración recibida por concepto de las labores que realiza dentro de la empresa; el $95 \%$ de los encuestados manifiestaron insatisfacción ante la retribución económica que reciben por su trabajo; esta situación es un motivo para mantener una sensación negativa de los beneficios que le otorga la profesión.

\section{Cuadro No 04:}

¿Cómo calificaría su desempeño laboral dentro de la empresa?

\begin{tabular}{|c|r|r|}
\hline Alternativas & \multicolumn{1}{|c|}{ fa } & \multicolumn{1}{|c|}{$\mathbf{f \%}$} \\
\hline Excelente & 12 & 30 \\
\hline Bueno & 28 & 70 \\
\hline Regular & 0 & 0 \\
\hline Deficiente & 0 & 0 \\
\hline Total & $\mathbf{4 0}$ & $\mathbf{1 0 0}$ \\
\hline
\end{tabular}

Fuente: Elaboración propia.

En la tabla se puede apreciar que existe un $70 \%$ de los contadores que considera que su desempeño laboral no es óptimo; de lo expuesto se puede deducir que la mayoría de los profesionales perciben que aún pueden mejorar su desempeño laboral en beneficio de la empresa, esta actitud debe relacionarse con la percepción que tienen los contadores de que su labor no está siendo bien remunerada.

\section{Cuadro No 05:}

¿Su título profesional lo adquirió en una universidad?

\begin{tabular}{|c|r|r|}
\hline Alternativas & \multicolumn{1}{|c|}{ fa } & \multicolumn{1}{|c|}{ f\% } \\
\hline Estatal & 32 & 80 \\
\hline Privada & 8 & 20 \\
\hline Total & $\mathbf{4 0}$ & $\mathbf{1 0 0}$ \\
\hline
\end{tabular}

Fuente: Elaboración propia.

El 80\% de los contadores tuvieron una formación profesional desarrollada en alguna de las universidades públicas existentes; el 20\% restante obtuvieron su título profesional en una universidad privada; los resultados indican que en la provincia de Manabí se mantiene una notable preferencia por el estudio superior en universidades del Estado; esta situación se debe a las facilidades económicas que tiene las universidades públicas para la obtención de títulos profesionales y al mejoramiento de la calidad periódica evidenciado por estas instituciones de estudios superiores durante los últimos años.

\section{Cuadro No 06:}

¿Cuando entró a trabajar, ¿se sintió totalmente capacitado para realizar las funciones que efectúa actualmente?

\begin{tabular}{|c|r|r|}
\hline Alternativas & \multicolumn{1}{|c|}{ Fa } & \multicolumn{1}{|c|}{ f\% } \\
\hline $\mathrm{Si}$ & 0 & 0 \\
\hline $\mathrm{No}$ & 40 & 100 \\
\hline Total & $\mathbf{4 0}$ & $\mathbf{1 0 0}$ \\
\hline
\end{tabular}

Fuente: Elaboración propia.

Las respuestas de los contadores sobre la seguridad que tuvieron al momento del inicio de sus funciones laborales como profesional dentro de las empresas son contundentes. El 100\% de los encuestados manifiestaron haberse sentido inseguros para realizar sus funciones como profesional en contabilidad en las empresas que los contrataron; a pesar de que este tipo de sensaciones es común en los profesionales que empiezan en nuevas actividades laborales, es un claro indicio de que los profesionales que salen de las universidades no están teniendo una formación que los acerque lo más posible al momento de iniciarse en puestos de trabajo relacionados con los conocimientos y habilidades adquiridas en la universidad; es decir que la institución no está efectuando simulacros sobre esta actividad inicial, misma que frecuentemente se convierte en un momento tenso para el profesional.

\section{Cuadro No 07:}

¿Considera que la universidad formó en usted todas las competencias necesarias para efectuar un eficiente desempeño laboral?

\begin{tabular}{|c|r|r|}
\hline Alternativas & \multicolumn{1}{|c|}{ fa } & \multicolumn{1}{|c|}{ f\% } \\
\hline $\mathrm{Si}$ & 0 & 0 \\
\hline $\mathrm{No}$ & 40 & 100 \\
\hline Total & $\mathbf{4 0}$ & $\mathbf{1 0 0}$ \\
\hline
\end{tabular}

Fuente: Elaboración propia. 
Mediante esta pregunta se pudo corroborar la inseguridad e insatisfacción que poseen los contadores respecto de la formación profesional recibida en las universidades, la opinión de desapruebo del $100 \%$ de los encuestados, se produce luego de que ellos han tenido una percepción real de las competencias que requiere para poder desempeñar adecuadamente las funciones asumidas en la empresa; por lo que, se constituye en una importante aportación para la evaluación de la pertinencia de la formación universitaria que se viene ofertando en las instituciones de educación superior de la provincia de Manabí.

\section{Cuadro No 08:}

¿Considera que la universidad formó en usted todas las competencias necesarias para efectuar un eficiente desempeño laboral?

\begin{tabular}{|c|r|r|}
\hline Alternativas & \multicolumn{1}{|c|}{ fa } & \multicolumn{1}{|c|}{ f\% } \\
\hline Conceptual & 0 & 0 \\
\hline Práctico & 16 & 40 \\
\hline Tecnológico & 24 & 60 \\
\hline Total & $\mathbf{4 0}$ & $\mathbf{1 0 0}$ \\
\hline
\end{tabular}

Fuente: Elaboración propia.

Los contadores opinaron que el elemento de desempeño laboral que menos se desarrolló en las universidades fue el uso de tecnología relacionada con el control contable, esto es, el uso de software contables que se aplican en las empresas para llevar el registro de la contabilidad de la organización y generar los informes respectivos que sirven de apoyo para la toma de decisiones gerenciales estratégicas; además los contadores consideraron que hay carencia de la parte práctica dentro de su formación universitaria; la información obtenida es coherente y coincide con la importancia que tiene la práctica y el conocimiento tecnológico para el ejercicio eficiente de las funciones profesionales requeridas.

\section{Cuadro No 09:}

¿Dónde aprendió más como profesional?

\begin{tabular}{|c|r|r|}
\hline Alternativas & \multicolumn{1}{|c|}{ fa } & \multicolumn{1}{|c|}{$\mathbf{f} \%$} \\
\hline Universidad & 0 & 0 \\
\hline Empresa & 40 & 100 \\
\hline Total & $\mathbf{4 0}$ & $\mathbf{1 0 0}$ \\
\hline
\end{tabular}

Fuente: Elaboración propia.
Los contadores en funciones activas laborales dentro de empresas privadas consideraron que el mayor porcentaje de aprendizaje que aplican actualmente en su gestión contable lo consiguieron en el ejercicio de sus funciones; esto significa que los profesionales le están dando mayor valor a lo que perciben de las actividades laborales que realizan y que se convierte en una valiosa experiencia profesional, y que desmerecen de alguna manera la capacitación que les brindó la universidad durante el proceso estudiantil.

\section{Cuadro No 10:}

¿Considera que las universidades se actualizan permanentemente para formar contadores plenamente capacitados para el ejercicio de sus funciones?

\begin{tabular}{|c|r|r|}
\hline Alternativas & \multicolumn{1}{|c|}{ fa } & \multicolumn{1}{|c|}{ f\% } \\
\hline $\mathrm{Si}$ & 0 & 0 \\
\hline $\mathrm{No}$ & 40 & 100 \\
\hline Total & $\mathbf{4 0}$ & $\mathbf{1 0 0}$ \\
\hline
\end{tabular}

Fuente: Elaboración propia.

Los contadores opinaron que las universidades no se están preparando adecuadamente para formar profesionales con un nivel de capacidades que exige el medio empresarial en el cual se desempeñaran los egresados; lo expuesto, aunque es algo subjetivo, significaría que la educación universitaria que reciben los profesionales en contabilidad dentro de la universidades de Manabí, no se está innovando en correlación directa con el avance de las exigencias laborales que mantienen las empresas.

\section{CONCLUSIONES}

1. Los contadores en funciones laborales activas de la provincia de Manabí consideran que la universidad donde se formaron profesionalmente, no los preparó adecuadamente para ejercer la gestión contable para la cual fueron contratados, lo cual provoca inseguridad en sus desempeños laborales, sobre todo durante los primeros días en los cuales tienen contacto con las actividades que deben realizar.

2. Los contadores consideran más importantes los conocimientos y habilidades que adquieren durante el ejercicio de sus funciones laborales profesionales, que aquellas que recibieron y fueron 
desarrolladas en las universidades. La percepción que tienen es un claro indicio de que las universidades no están ejecutando una eficiente formación en los contadores respecto al desempeño de los mismos en la vida real.

3. Los contadores consideran que el elemento laboral que utilizan en sus funciones y que no se trabajo adecuadamente en las universidades es el manejo de programas contables que utilizan las empresas privadas contemporáneas; además opinan que las universidades no los acerca a la realidad laboral mediante el análisis y desarrollo de ejemplos prácticos y reales relacionados con el entorno laboral en el cual tienen que desenvolverse posteriormente.

4. Los contadores creen que el estudio en las universidades no está evolucionando al mismo ritmo de las exigencias competitivas laborales que demandan las empresas privadas de la mano de obra calificada que desempeña gestiones dentro de ellas.

\section{REFERENCIAS BIBLIOGRÁFICAS}

1. ARAUJO, M.y GUERRA, M. (2007). Inteligencia emocional y desempeño laboral en las instituciones de educación superior públicas. CICAG. Volumen IV. 2da. Edición.
2. BOHÓRQUEZ, B. y VÁZQUEZ, D. (2002): Estudio comparativo de la satisfacción del cliente interno con respecto a la calidad del servicio de la gestión de Recursos Humanos en dos empresas de trabajo temporal, ubicadas en el área metropolitana de Caracas. Relaciones Industriales. Universidad Católica Andrés Bello, Caracas.

3. CHIAVENATO,I.(2002). Comportamiento organizacional. McGraw-Hill / Interamericana editores, S.A. de C.V. Segunda edición. México.

4. Federación Nacional de Contadores del Ecuador (FNCE, 1991). Código de Ética del Contador.

5. GARCÏA,J.y CASANUEVA, C.(2001). Prácticas de la gestión empresarial. Madrid: Mc Graw Hill.

6. MONTANER, J. y PEROZO, J. (2008). Formación ética del Contador Público. Caso: Facultad de Ciencias Económicas y Sociales de la Universidad del Zulia. Venezuela.

7. PELIKAN, J., (1992). The idea of the university. New Haven, Connecticut: Yale University Press.

8. STONER, J. (1994). Administración. 5ta. Edición. Editorial Prentice - Hall Hispanoamericana S.A.

9. VALLAEYS, F. (2008) ¿Qué es la Responsabilidad Social Universitaria?. Pontificia Universidad Católica del Perú. 